\title{
Implantation of autologous peripheral nerve grafts into the substantia nigra of subjects with idiopathic Parkinson's disease treated with bilateral STN DBS: a report of safety and feasibility
}

\author{
Craig G. van Horne, MD, PhD, ${ }^{1-3}$ Jorge E. Quintero, PhD, ${ }^{1,3}$ Julie A. Gurwell, PA-C, PhD,1,4 \\ Renee P. Wagner, RN, ${ }^{4}$ John T. Slevin, MD, MBA, ${ }^{1,4}$ and Greg A. Gerhardt, PhD' ${ }^{1-4}$
}

${ }^{1}$ Brain Restoration Center, and Departments of ${ }^{2}$ Neurosurgery ${ }^{3}$ Anatomy \& Neurobiology, and ${ }^{4}$ Neurology, University of Kentucky, Lexington, Kentucky

OBJECTIVE One avenue of intense efforts to treat Parkinson's disease (PD) involves the delivery of neurotrophic factors to restore dopaminergic cell function. A source of neurotrophic factors that could be used is the Schwann cell from the peripheral nervous system. The authors have begun an open-label safety study to examine the safety and feasibility of implanting an autologous peripheral nerve graft into the substantia nigra of PD patients undergoing deep brain stimulation (DBS) surgery.

METHODS Multistage DBS surgery targeting the subthalamic nucleus was performed using standard procedures in 8 study participants. After the DBS leads were implanted, a section of sural nerve containing Schwann cells was excised and unilaterally delivered into the area of the substantia nigra. Adverse events were continuously monitored.

RESULTS Eight of 8 participants were implanted with DBS systems and grafts. Adverse event profiles were comparable to those of standard DBS surgery. Postoperative MR images did not reveal edema, hemorrhage, or significant signal changes in the graft target region. Three participants reported a patch of numbness on the outside of the foot below the sural nerve harvest site.

CONCLUSIONS Based on the safety outcome of the procedure, targeted peripheral nerve graft delivery to the substantia nigra at the time of DBS surgery is feasible and may provide a means to deliver neurorestorative therapy.

Clinical trial registration no.: NCT01833364 (clinicaltrials.gov)

https://thejns.org/doi/abs/10.3171/2016.2.JNS151988

KEY WORDS deep brain stimulation; biological therapy; multimodal DBS; neurotrophic factor; functional neurosurgery; peripheral nerve

$\mathrm{P}$ ARKINSON's disease (PD) is a complex, chronic, neurodegenerative disorder with motor symptoms produced by the progressive degeneration of dopaminergic neurons within the substantia nigra pars compacta in the midbrain. While existing pharmaceutical and surgical therapies have some impact on the symptoms of PD, there is no current therapy that stops or reverses the ongoing loss of cell function, deterioration, and ultimately cell death. Early preventative intervention is difficult because symptoms of PD do not begin until $75 \%-80 \%$ of the dopaminergic neurons have lost measurable function. ${ }^{2} \mathrm{How}-$ ever, animal studies show that a portion of damaged cells in the substantia nigra may still be able to display a level of functional recovery. ${ }^{9}$
The 2 main strategies aimed at preventing disease progression have been to either transplant cells or tissues in an attempt to replace the lost dopaminergic neurons or to deliver growth factors that could restore the injured neurons before they die. Clinical trials involving the transplantation of dopaminergic tissues into the brain have not been able to demonstrate clinical benefit. ${ }^{20}$ Additionally, the source of the transplant material, fetal tissue, has raised considerable ethical issues regarding the viability of this therapeutic strategy. ${ }^{14}$ On the other hand, studies involving the application of various growth factors to dopaminergic tissues have shown more promising results. Preclinical studies have identified several growth factors, including glial-derived neurotrophic factor (GDNF), ${ }^{9}$ brain-derived

ABBREVIATIONS BDNF = brain-derived neurotrophic factor; CRW = Cosman-Roberts-Wells; DBS = deep brain stimulation; GDNF = glial-derived neurotrophic factor; NGF = nerve growth factor; PD = Parkinson's disease; PDQ-8 = Parkinson's Disease Questionnaire; STN = subthalamic nucleus; UPDRS = Unified Parkinson's Disease Rating Scale.

SUBMITTED August 27, 2015. ACCEPTED February 19, 2016

INCLUDE WHEN CITING Published online May 6, 2016; DOI: 10.3171/2016.2.JNS151988. 
neurotrophic factor (BDNF), ${ }^{30}$ and ciliary neurotrophic factor $(\mathrm{CNTF}),{ }^{4}$ that are able to support the survival and maintenance of dopaminergic neurons. Additionally, pilot studies investigating the direct delivery of GDNF into the brains of patients with PD have shown safety as well as some clinical improvement. ${ }^{10,23,28}$ Other clinical trials have also sought to deliver neurotrophic support, including neurturin, ${ }^{18,19}$ to patients with PD. Nevertheless, strategies utilizing growth factor delivery raise additional questions, such as which growth factors should be used and how should they be obtained, delivered, and dosed (see Rodrigues et al. ${ }^{25}$ ). Currently purified growth factors are very difficult to manufacture, and access is limited by substantial patent restrictions. As a way to overcome these obstacles, we designed an open-label safety study to investigate the use of an autologous source of cellular tissue from the peripheral nervous system, the Schwann cell, which has been shown to produce and express several important growth factors, including GDNF, ${ }^{29} \mathrm{BDNF},{ }^{1}$ and neurotrophin-3 (NT-3) ${ }^{13}$ Peripheral nerve grafts and purified Schwann cell transplants into the CNS have been extensively employed in animal and human studies. . $^{3,6,11,15 \text {, }}$ 17,27,33,34 Furthermore, in rodents and primates, peripheral nerve grafts and Schwann cell transplants survive and release nerve growth factor (NGF) even up to 3 months after delivery. ${ }^{16,24}$

The central hypothesis of this study is that the harvesting of the peripheral nerve graft will initiate the production of growth factors through the injury response and that the transplanted graft will allow the Schwann cells to associate with the degenerating dopaminergic neurons and deliver the expressed growth factors to restore and maintain the injured neurons over time. In this paper we describe the delivery of a peripheral nerve graft to the substantia nigra and the subsequent safety and adverse event profile of the procedure.

We chose to investigate the safety and tolerability of peripheral nerve grafts in participants who had been selected clinically as good candidates for deep brain stimulation (DBS) surgery. There are several advantages to this approach. First, participants received the full benefit of the DBS therapy. The implant procedure and follow-up design was purposefully designed to not interfere with the DBS clinical application. Second, the graft implantation was performed at the time of DBS surgery and therefore did not require an additional surgical intervention. Third, DBS therapy was amenable to testing the effects of an intervention on the baseline components of PD. Although this approach has been proposed previously for investigating a gene therapy approach,, 26 it was not actually performed. Thus we report the first clinical study to involve the investigation of a surgical therapeutic intervention in combination with DBS therapy. Here we describe the surgical details, graft implant technique, and perioperative adverse event profile.

\section{Methods}

\section{Clinical Trial Design}

This study was designed as a 12-month, open-label, single-center, Phase I trial to assess safety and feasibility as the primary endpoints. It was registered with the ClinicalTrials.gov database (http://clinicaltrials.gov), and its registration number is NCT01833364. Additional efficacy data were collected and will be reported in a later, separate paper. The timeline of the trial design is shown in Fig. 1. The study was approved by the University of Kentucky institutional review board, and informed consent was obtained from all study participants.

\section{Patient Selection}

Eight patients with a diagnosis of idiopathic PD, who had been selected and had provided consent for DBS of the subthalamic nucleus (STN), were subsequently informed about the study and then agreed to participate in the study and gave formal consent. Thus, agreeing to participate in the study had no influence on the decision to undergo DBS therapy. Inclusion criteria included an age of 40 to 75 years, symptomatic, idiopathic PD for longer than 5 years, and an ability to participate in all follow-up appointments. As part of the standard preoperative DBS protocol, participants were evaluated using the Unified Parkinson's Disease Rating Scale (UPDRS), both on and off medication, and underwent a formal neuropsychological evaluation. Preoperative imaging consisted of an MRI study for targeting purposes.

\section{Safety and Feasibility}

Participants were monitored for both perioperative and long-term postoperative adverse events. Clinical monitoring for this report includes the perioperative time points, the 1-month study evaluation visit, and all clinic visits in between. During this time period, adverse events were recorded from clinical reporting, chart review, and specific queries during the clinical visits. A postoperative 1.5-T MRI study was performed within 48 hours of the implantation surgery. Adverse events were categorized as either related to the graft or graft procedure, DBS surgery or therapy, or not related to either surgical or therapeutic component. In addition, adverse events were categorized as mild, moderate, or severe, as defined in the Medical Dictionary for Regulatory Activities, version 11.0. Serious adverse events were designated as any event that resulted in death, disability, or prolonged or new hospitalization or that was life threatening or that required medical or surgical intervention.

\section{Surgical Implantation}

Participants were evaluated preoperatively with a targeting 1.5- or 3.0-T MRI. The cortical entry points, trajectories, and STN were targeted bilaterally using Brainlab software (IPlan 3.0 Stereotaxy). The graft cannula trajectory was calculated as a trajectory parallel to the stimulating lead trajectory. The DBS implantation was performed in 2 stages. The traditional staging of the surgeries was reversed ${ }^{31}$ by first implanting the pulse generator and lead extensions and placing the bur holes, based on frameless neuronavigation (Brainlab), during general anesthesia. Once this was completed, the patients were repositioned for access to the sural nerve. Through a standard sural nerve biopsy incision, the sural nerve was exposed and 


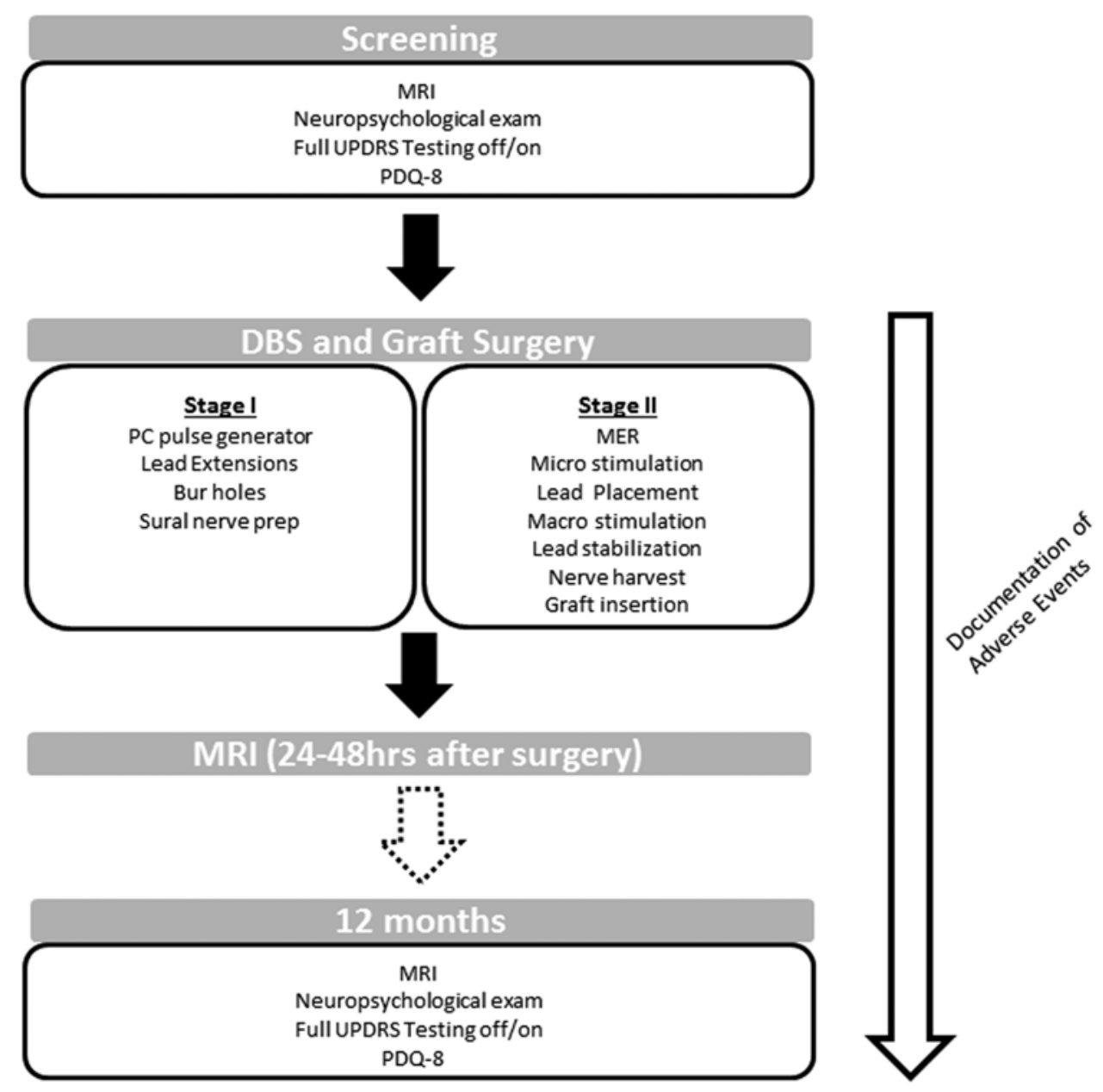

FIG. 1. Schematic time line of study design. MER = microelectrode recording; PC pulse generator = Activa PC pulse generator.

transected proximally. Two silk ties were loosely secured to the proximal and distal end of the nerve, distal to the transection. A $1-\mathrm{cm}$ tail was left on the ties, providing easy identification of the nerve during the harvesting procedure, which was performed during the second stage of the surgery, 3-5 days later. The incision was then closed.

The second stage was completed 3 to 5 days after the first. Participants were fitted with an Integra CRW (Cosman-Roberts-Wells) frame and localizer and a thin-cut CT scan was performed for targeting. Frame coordinates for the targets and trajectories were calculated based on image fusion sequences using IPlan 3.0 Stereotaxy (Brainlab). Initial STN targets were chosen visually based on susceptibility-weighted imaging sequences. Microelectrode recordings were performed using a single electrode. The inferior border of the STN was designated as the electrophysiological point where cells were no longer identified and the increase in baseline that is typically seen in the STN dropped back to background levels. In some cases, cells with profiles consistent with substantia nigra pars reticulata were identified. Stimulating electrodes (Medtronic, 3387) were placed with the distal contact at the ventral border of the STN. Electrode contacts were then tested for efficacy and side effects. The STN contra- lateral to the least symptomatic side was implanted first. Once testing was complete, the bur hole was filled with Hydroset (Stryker) and the electrode was secured to the skull, connected to the lead extension, and internalized.

After testing of the second electrode was complete, the electrode was secured to the skull using a mini-plate. The graft target zone was then calculated through a trajectory parallel to the stimulating electrode and approximately 3 $\mathrm{mm}$ posterior and lateral. A guide cannula (FHC Inc., 1.8$\mathrm{mm}$ outer diameter) was then placed to target within the graft target zone. The target zone was designated to occupy a 5-mm space within the substantia nigra pars reticulata between $1 \mathrm{~mm}$ and $6 \mathrm{~mm}$ deep to the inferior border of the STN (Fig. 2). The sural nerve incision was prepared and opened. The sural nerve was identified using the silk ties, and a 2-cm segment of nerve was harvested distal to the previous transection site. The nerve segment was then rinsed in sterile saline in a petri dish. The epineurium was removed sharply. The remaining fascicles were then cut transversely to produce a number of smaller segments approximately $1 \mathrm{~mm}$ in length. Five to six segments less than $1.5 \mathrm{~mm}$ in diameter were then loaded into the custom-made graft cannula through a side loading port and advanced to the distal end of the graft cannula using the flat-ended 


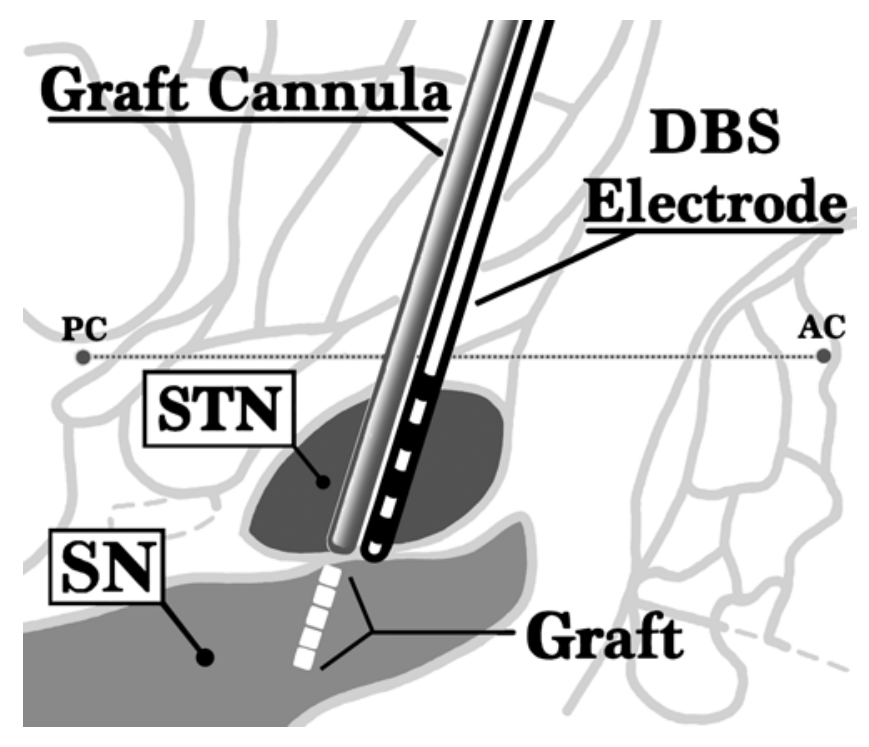

FIG. 2. Graft delivery was targeted to the substantia nigra at 1-6 mm below the base of the STN where the DBS leads were implanted. The anterior commissure-posterior commissure line is shown. $\mathrm{AC}=$ anterior commissure; $\mathrm{PC}=$ posterior commissure; $\mathrm{SN}=$ substantia nigra.

inner stylet (Fig. 3). The stylet was then locked in place with set screws. The guide cannula was removed, and the graft cannula was placed to target through the same bur hole used for the stimulating electrode. The graft was deployed by releasing the inferior set screw and retracting the graft cannula $5 \mathrm{~mm}$. After 2 minutes, the assembly was rotated in place several times to facilitate graft detachment from the assembly. The graft cannula assembly was then removed. The dura was covered with Durepair dura regeneration matrix (Medtronic) and the bur hole was filled with HydroSet. This sequencing was chosen so that the grafting procedure would not interfere with the DBS implantation.

\section{Graft Cannula}

The graft cannula design was based on a custom design modification of a standard guide cannula (lead insertion tube, FHC). The modifications included a side window proximal to the distal tip, an extended length to reach 6 $\mathrm{mm}$ below target for CRW specifications, and a modified, 2-set-screw locking coupler to stabilize the inner stylet with the outer cannula. This construction allows for the graft tissue to be deposited into brain parenchyma rather than being pushed in. The dimensions of the graft cannula are shown in Fig. 3 (upper).

\section{Postoperative Evaluation}

After DBS and graft implantation, participants were admitted overnight and received a postoperative MRI to confirm electrode placement and to evaluate the region of the graft target zone. Additionally, stimulator programming was initiated during this time period. Participants were then followed clinically through routine postoperative visits, programming visits, and for their 1-month postoperative study visits. While the protocol includes additional study visits at 6,9 , and 12 months, this mid-study

\section{Graft Cannula Tip}

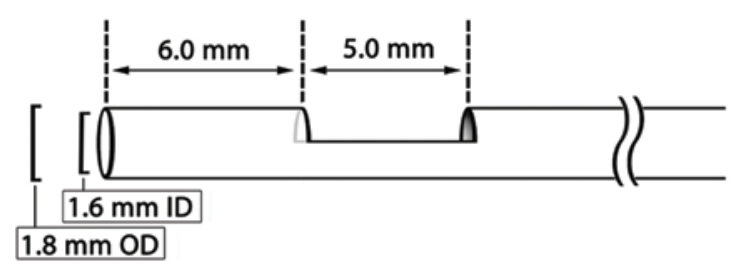

\section{Inner Stylet}
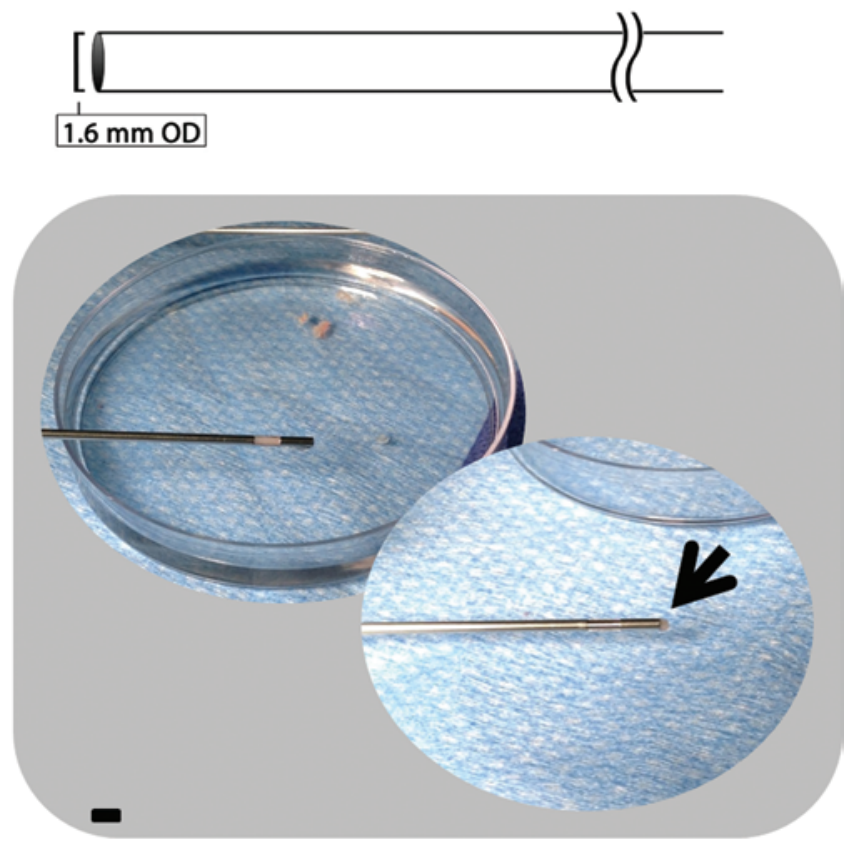

FIG. 3. Upper: Schematic illustration showing the graft cannula specifications. The window at the tip of the cannula is used for loading tissue before implantation and delivery to the target. ID = inner diameter; OD = outer diameter. Lower: Photograph of the harvested sural nerve fascicles. Autologous sural nerve fascicles were harvested, identified, and loaded in the side window of the cannula port and then primed for delivery. The arrow points to the location in a loaded cannula where the graft tissue extends beyond the cannula opening. Bar $=5 \mathrm{~mm}$. Figure is available in color online only.

report focuses on the safety data through the 1st month time point for all 8 patients.

\section{Results}

\section{Participant Profile}

Eight participants (6 male and 2 female) were enrolled in the study (Table 1). Neuropsychological evaluations demonstrated mild to moderate declines consistent with PD related changes and did not demonstrate any significant deterioration that would preclude them from the study.

\section{Surgical Results}

All 8 participants were successfully implanted with bi- 
TABLE 1. Baseline characteristics of the 8 study participants

\begin{tabular}{cc}
\hline \multicolumn{1}{c}{ Characteristic } & Mean \pm SD \\
\hline Age in years & $62.9 \pm 9.2$ \\
\hline PD duration in years & $9.8 \pm 3.9$ \\
\hline L-Dopa equivalents* in mg/day & $854 \pm 556$ \\
\hline UPDRS score & \\
\hline Part III (motor symptoms) & $32.5 \pm 9.7$ \\
\hline OFF & $13.4 \pm 9.7$ \\
\hline ON & $38.4 \pm 13.1$ \\
\hline Total ON & $9.4 \pm 4.4$ \\
\hline PDQ-8
\end{tabular}

* As described by Pahwa et al. ${ }^{21}$

lateral DBS electrodes and underwent the grafting procedure without any technical difficulties. The mean duration of time for bilateral DBS electrode and graft placement was 3 hours 51 minutes (SD 26 minutes), which is consistent with our previously reported surgery times. ${ }^{31}$

Postoperative MR images showed accurate placements of all 16 DBS electrodes. There was no evidence of hemorrhage or edema around the electrodes. With respect to the graft target zone, there was no significant signal variability providing evidence for graft placement confirmation, significant edema, or hemorrhage (Fig. 4).

\section{Adverse Events}

Reported adverse events (Table 2) were in line with profiles from DBS surgery. ${ }^{22,32}$ After the second stage of the surgery, 3 participants described a patch of numbness on the outer aspect of the foot.

\section{Discussion}

The primary objectives of this pilot study were to assess safety and feasibility of the grafting procedure along with tolerability of the graft. Although the concept of tissue implantation into the brain is not new, the issue of safety is critical in that this is the first clinical trial where grafts have been implanted in conjunction with DBS therapy.

With respect to the grafting procedure, we show that peripheral nerve tissue can be readily harvested, loaded into a cannula, and stereotactically deposited into the target site, with the procedure adding only 30-60 minutes. Concerning safety, none of the adverse events were attributable to the grafting procedure except for mild, transient numbness on the outer aspect of the foot where the sural nerve biopsy occurred. The adverse event profile is not different from that of nongrafting DBS procedures performed in our center (unpublished observations) or published reports. ${ }^{12,22,32}$ With a cannula of a standard $(1.8-\mathrm{mm})$ size, the overall risk of this procedure, compared with DBS surgery alone, can be estimated to be that of a single additional

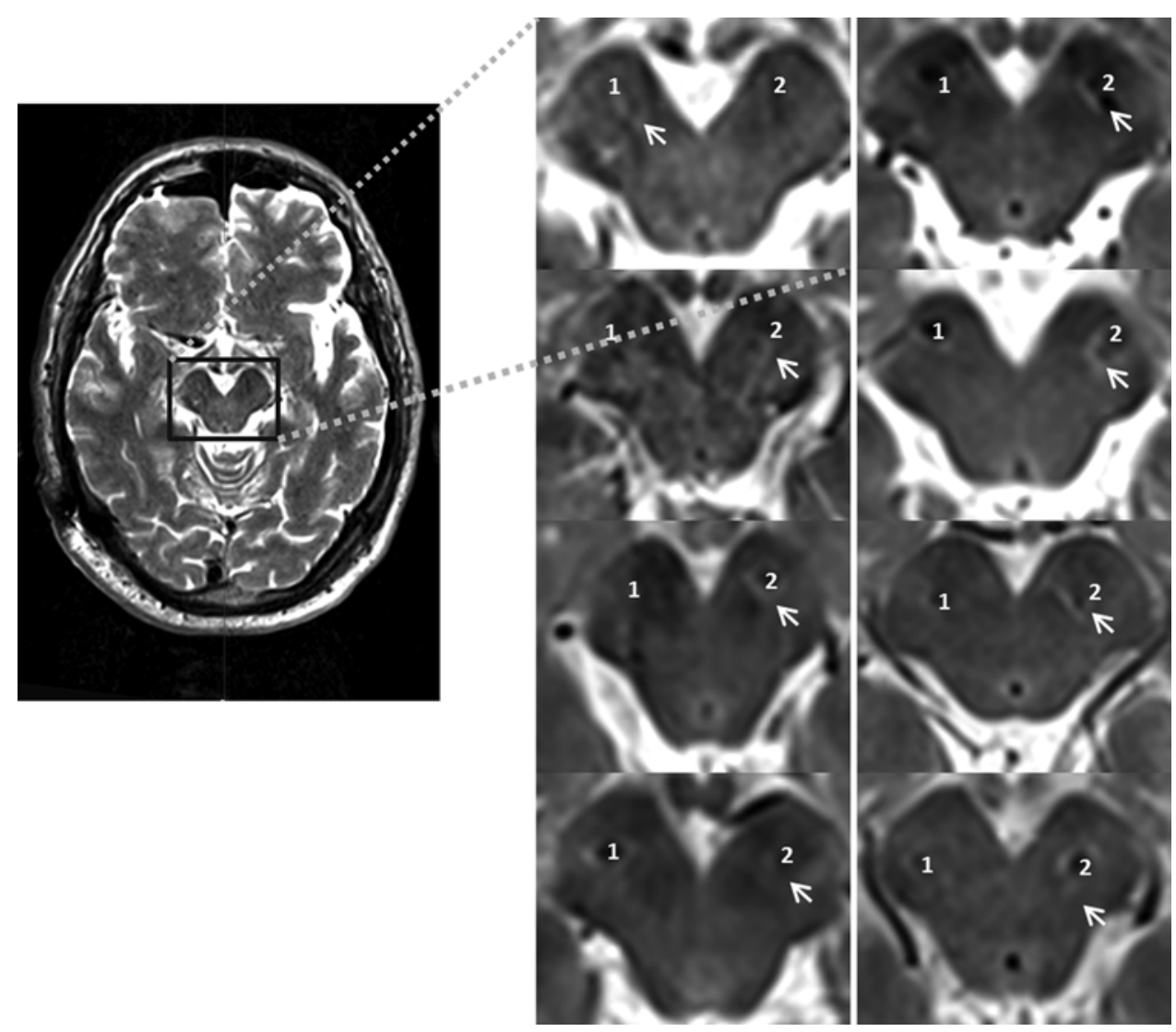

FIG. 4. Postoperative axial T2-weighted MR images obtained in the 8 participants showing the area targeted for peripheral nerve graft implantation. The arrows indicate the area targeted for graft delivery; 1 and 2 indicate DBS leads. 
TABLE 2. One-month-postsurgery adverse event profile

\begin{tabular}{clll}
\hline $\begin{array}{c}\text { Participant } \\
\text { No. }\end{array}$ & Adverse Event & Status & \multicolumn{1}{c}{ Relatedness } \\
\hline 1 & Urinary retention & Resolved & Surgical procedure \\
\hline 2 & $\begin{array}{l}\text { Hypomania } \\
\text { Shortness of breath }\end{array}$ & $\begin{array}{l}\text { Resolved } \\
\text { Resolved }\end{array}$ & $\begin{array}{l}\text { Stimulation } \\
\text { Not related }\end{array}$ \\
\hline 3 & None & NA & NA \\
\hline 4 & Superficial cellulitis & Resolved & Surgical procedure \\
\hline 5 & Cough & Resolved & Surgical procedure \\
& Headache & Resolved & \\
\hline 6 & None & NA & NA \\
\hline 7 & None & NA & NA \\
\hline 8 & None & NA & NA \\
\hline
\end{tabular}

$\mathrm{NA}=$ not applicable.

cannula pass. Based on these findings, the results from this study provide direct evidence that graft implantation can be carried out at the same time as DBS implantation without significant risk to the recipient.

There are several advantages to combining nerve graft delivery at the time of DBS surgery when performed as described here: 1) because the grafting procedure is performed after the implantation of the pulse generator, lead extensions, and DBS leads, the grafting procedure does not interfere with the DBS implantation procedure; 2) an additional surgery outside of the scheduled DBS procedures is not required; 3) participants supply their own tissue, which eliminates the potential for immune rejection that is present with other transplantation strategies. Because the grafting procedure used autologous grafts harvested and delivered during the same surgical procedure and the tissue was not chemically or genetically modified, the study did not have to comply with the requirements of the FDA regulations on tissue delivery.

Although the placement of the DBS electrodes could be confirmed with postoperative MRI, we found no significant or consistent MRI signal changes within the graft target region. This finding was somewhat unexpected in that we anticipated that the insertion of the cannula, in and of itself, into the region would lead to a consistent, identifiable increase in T2 signal on the MRI. One possible explanation is that the implanted graft, being solid tissue, negates the potential T2 signal change. Nevertheless, this grafting approach combined with DBS does present a limit on imaging with MR, as currently only 1.5-T MR images can be obtained with implanted DBS electrodes. Furthermore, while sural nerve grafts to the basal ganglia in nonhuman primates show extensive NGF-reactive Schwann cell survival 3 months after transplantation,,$^{16}$ because the peripheral nerve tissue is not biologically or chemically modified, noninvasive tracking of graft survival is not possible. Assessing the viability of the graft over time remains one of the key limitations of this technique. For now, our approach to begin addressing issues of graft viability, factors that may enhance the viability, and functional assessments of the graft is to use animal studies to model the graft delivery and implantation. Currently, we lack a pro- file of the neurotrophic factors that are present in the sural nerve that is being implanted. To address this gap, we plan on employing proteomics to determine which neurotrophic factors may be present in harvested sural nerves. In the clinical domain, clinical outcome assessments will be the standard for measuring the success of the technique, while future studies may use other imaging modalities, such as PET, to further investigate the ability to track graft placement and/or function in vivo.

We chose the substantia nigra as our target for graft placement for several reasons. In addition to being the anatomical location of the dopaminergic neuron cell bodies, the substantia nigra is anatomically close to the STN, and this makes it an ideal target for minimizing risk following the DBS procedure. On the entry side, the substantia nigra as a target allowed us to use the same bur hole, without expansion, to target the STN for DBS lead implantation and the substantia nigra for graft placement. The dorsal border of the substantia nigra pars reticulata was identified based on preoperative MR targeting and the results of microelectrode recordings, but given that the cannula delivered the graft across a 6-mm span, it is possible that other regions of the substantia nigra were also reached. In $\mathrm{PD}$, more cells are spared in the area of the pars reticulata than in the pars compacta, ${ }^{5,8}$ thus providing neurotrophic support to cells that may be spared or injured rather than cells that are dead could be a strategy for therapy.

While we have focused on the safety and feasibility of the operative and perioperative aspects of the technique in this report, we do think longer-term studies will be a necessary step on the way to fully establishing the safety of the procedure. We are continuing to collect safety data, which we plan to present in conjunction with the longerterm clinical outcome aspect of the study.

The strategy of combining the delivery of biological therapy with DBS surgery has been recently gaining momentum. ${ }^{26}$ DBS therapy alone is insufficient for treating all of the symptoms associated with PD, and so, new therapies need to be developed to enhance the therapeutic benefits of DBS. The combined strategy outlined here presents one means of fulfilling this goal. In terms of clinical trial design, this strategy has the advantage of obviating the need for a sham surgical procedure while lowering trial-associated costs, since DBS surgery is a reimbursable procedure. The results from the delivery of peripheral nerve graft into the midbrain shown here may usher in a new approach for combining biologicals with DBS to provide multimodal therapy for PD.

\section{Conclusions}

We have accomplished our primary objectives of demonstrating the safety and feasibility of implanting peripheral nerve tissue in participants who are undergoing DBS surgery and treatment. We feel that this is an important, critical step as the first clinical study to combine the 2 approaches. However, we remain cautious, as the longer-term effects and safety of this combined procedure need to be further examined over the course of years, and of course, in a larger population. Thus far, the results provide ini- 
tial evidence that patients who elect to receive DBS therapy can be good candidates for neurorestorative therapies requiring invasive deployment, such as peripheral nerve grafts and in the future stem cell grafting, growth factor delivery, or gene therapy.

\section{Acknowledgments}

This work was supported by gifts to the Brain Restoration Center; Tom Dupree for Parkinson's Disease Research; Pro's Players Fore Parkinson's; University of Kentucky start-up funds (to Dr. van Horne); and the National Center for Advancing Translational Sciences, through NIH grant UL1TR000117. The content is solely the responsibility of the authors and does not necessarily represent the official views of the NIH.

\section{References}

1. Acheson A, Barker PA, Alderson RF, Miller FD, Murphy RA: Detection of brain-derived neurotrophic factor-like activity in fibroblasts and Schwann cells: inhibition by antibodies to NGF. Neuron 7:265-275, 1991

2. Bernheimer H, Birkmayer W, Hornykiewicz O, Jellinger $\mathrm{K}$, Seitelberger F: Brain dopamine and the syndromes of Parkinson and Huntington. Clinical, morphological and neurochemical correlations. J Neurol Sci 20:415-455, 1973

3. Bunge MB, Wood PM: Realizing the maximum potential of Schwann cells to promote recovery from spinal cord injury. Handb Clin Neurol 109:523-540, 2012

4. Clatterbuck RE, Price DL, Koliatsos VE: Ciliary neurotrophic factor prevents retrograde neuronal death in the adult central nervous system. Proc Natl Acad Sci U S A 90:2222-2226, 1993

5. Damier P, Hirsch EC, Agid Y, Graybiel AM: The substantia nigra of the human brain. II. Patterns of loss of dopaminecontaining neurons in Parkinson's disease. Brain 122:14371448, 1999

6. Deng LX, Walker C, Xu XM: Schwann cell transplantation and descending propriospinal regeneration after spinal cord injury. Brain Res 1619:104-114, 2015

7. During MJ, Kaplitt MG, Stern MB, Eidelberg D: Subthalamic GAD gene transfer in Parkinson disease patients who are candidates for deep brain stimulation. Hum Gene Ther 12:1589-1591, 2001

8. Fearnley JM, Lees AJ: Ageing and Parkinson's disease: substantia nigra regional selectivity. Brain 114:2283-2301, 1991

9. Gash DM, Zhang Z, Ovadia A, Cass WA, Yi A, Simmerman L, et al: Functional recovery in parkinsonian monkeys treated with GDNF. Nature 380:252-255, 1996

10. Gill SS, Patel NK, Hotton GR, O'Sullivan K, McCarter R, Bunnage M, et al: Direct brain infusion of glial cell linederived neurotrophic factor in Parkinson disease. Nat Med 9:589-595, 2003

11. Guest J, Santamaria AJ, Benavides FD: Clinical translation of autologous Schwann cell transplantation for the treatment of spinal cord injury. Curr Opin Organ Transplant 18:682-689, 2013

12. Hariz MI, Rehncrona S, Quinn NP, Speelman JD, Wensing C: Multicenter study on deep brain stimulation in Parkinson's disease: an independent assessment of reported adverse events at 4 years. Mov Disord 23:416-421, 2008

13. Heumann R, Korsching S, Bandtlow C, Thoenen H: Changes of nerve growth factor synthesis in nonneuronal cells in response to sciatic nerve transection. J Cell Biol 104:1623 1631,1987

14. Hoffer BJ, Olson L: Ethical issues in brain-cell transplantation. Trends Neurosci 14:384-388, 1991

15. Kohama I, Lankford KL, Preiningerova J, White FA, Vollmer TL, Kocsis JD: Transplantation of cryopreserved adult human Schwann cells enhances axonal conduction in demyelinated spinal cord. J Neurosci 21:944-950, 2001

16. Kordower JH, Fiandaca MS, Notter MF, Hansen JT, Gash DM: NGF-like trophic support from peripheral nerve for grafted rhesus adrenal chromaffin cells. J Neurosurg 73:418-428, 1990

17. Levi AD, Dancausse H, Li X, Duncan S, Horkey L, Oliviera M: Peripheral nerve grafts promoting central nervous system regeneration after spinal cord injury in the primate. $\mathbf{J}$ Neurosurg 96 (2 Suppl):197-205, 2002

18. Marks WJ Jr, Bartus RT, Siffert J, Davis CS, Lozano A, Boulis N, et al: Gene delivery of AAV2-neurturin for Parkinson's disease: a double-blind, randomised, controlled trial. Lancet Neurol 9:1164-1172, 2010

19. Marks WJ Jr, Ostrem JL, Verhagen L, Starr PA, Larson BS, Bakay RA: Safety and tolerability of intraputaminal delivery of CERE-120 (adeno-associated virus serotype 2-neurturin) to patients with idiopathic Parkinson's disease: an open-label, phase I trial. Lancet Neurol 7:400-408, 2008

20. Olanow CW, Goetz CG, Kordower JH, Stoessl AJ, Sossi V, Brin MF, et al: A double-blind controlled trial of bilateral fetal nigral transplantation in Parkinson's disease. Ann Neurol 54:403-414, 2003

21. Pahwa R, Wilkinson SB, Overman J, Lyons KE: Bilateral subthalamic stimulation in patients with Parkinson disease: long-term follow up. J Neurosurg 99:71-77, 2003

22. Patel DM, Walker HC, Brooks R, Omar N, Ditty B, Guthrie BL: Adverse events associated with deep brain stimulation for movement disorders: analysis of 510 consecutive cases. Neurosurgery 11 (Suppl 2):190-199, 2015

23. Patel NK, Bunnage M, Plaha P, Svendsen CN, Heywood P, Gill SS: Intraputamenal infusion of glial cell line-derived neurotrophic factor in PD: a two-year outcome study. Ann Neurol 57:298-302, 2005

24. Pizzorusso T, Fagiolini M, Fabris M, Ferrari G, Maffei $\mathrm{L}$ : Schwann cells transplanted in the lateral ventricles prevent the functional and anatomical effects of monocular deprivation in the rat. Proc Natl Acad Sci U S A 91:25722576, 1994

25. Rodrigues TM, Jerónimo-Santos A, Outeiro TF, Sebastião AM, Diógenes MJ: Challenges and promises in the development of neurotrophic factor-based therapies for Parkinson's disease. Drugs Aging 31:239-261, 2014

26. Rowland NC, Starr PA, Larson PS, Ostrem JL, Marks WJ Jr, Lim DA: Combining cell transplants or gene therapy with deep brain stimulation for Parkinson's disease. Mov Disord 30:190-195, 2015

27. Saberi H, Moshayedi P, Aghayan HR, Arjmand B, Hosseini SK, Emami-Razavi SH, et al: Treatment of chronic thoracic spinal cord injury patients with autologous Schwann cell transplantation: an interim report on safety considerations and possible outcomes. Neurosci Lett 443:46-50, 2008

28. Slevin JT, Gerhardt GA, Smith CD, Gash DM, Kryscio $\mathrm{R}$, Young B: Improvement of bilateral motor functions in patients with Parkinson disease through the unilateral intraputaminal infusion of glial cell line-derived neurotrophic factor. J Neurosurg 102:216-222, 2005

29. Springer JE, Mu X, Bergmann LW, Trojanowski JQ: Expression of GDNF mRNA in rat and human nervous tissue. Exp Neurol 127:167-170, 1994

30. Tsukahara T, Takeda M, Shimohama S, Ohara O, Hashimoto $\mathrm{N}$ : Effects of brain-derived neurotrophic factor on 1-methyl4-phenyl-1,2,3,6-tetrahydropyridine-induced parkinsonism in monkeys. Neurosurgery 37:733-741, 1995 
31. van Horne CG, Vaughan SW, Massari C, Bennett M, Asfahani WS, Quintero JE, et al: Streamlining deep brain stimulation surgery by reversing the staging order. $\mathbf{J}$ Neurosurg 122:1042-1047, 2015

32. Videnovic A, Metman LV: Deep brain stimulation for Parkinson's disease: prevalence of adverse events and need for standardized reporting. Mov Disord 23:343-349, 2008

33. Watts RL, Mandir AS, Bakay RA: Intrastriatal cografts of autologous adrenal medulla and sural nerve in MPTPinduced parkinsonian macaques: behavioral and anatomical assessment. Cell Transplant 4:27-38, 1995

34. Watts RL, Subramanian T, Freeman A, Goetz CG, Penn RD, Stebbins GT, et al: Effect of stereotaxic intrastriatal cografts of autologous adrenal medulla and peripheral nerve in Parkinson's disease: two-year follow-up study. Exp Neurol 147:510-517, 1997

\section{Disclosures}

Dr. van Horne and Dr. Slevin have received educational support grants from Medtronic. Dr. Gerhardt has previously received research support from Medtronic.

\section{Author Contributions}

Conception and design: van Horne. Acquisition of data: van Horne, Quintero, Gurwell, Wagner, Slevin. Analysis and interpretation of data: van Horne, Quintero, Slevin, Gerhardt. Drafting the article: van Horne, Quintero. Critically revising the article: van Horne, Gerhardt. Reviewed submitted version of manuscript: van Horne, Quintero, Gurwell, Slevin, Gerhardt. Approved the final version of the manuscript on behalf of all authors: van Horne. Statistical analysis: Quintero. Administrative/technical/ material support: Wagner.

\section{Correspondence}

Craig G. van Horne, Department of Neurosurgery, University of Kentucky, 800 Rose St., Lexington, KY 40536. email: craigvanhorne@uky.edu. 